\title{
Potential of PET/CT in assessing dementias with emphasis on cerebrovascular disorders
}

\author{
Austin J. Borja ${ }^{1,2} \cdot$ Emily C. Hancin ${ }^{1,3} \cdot$ Vincent Zhang ${ }^{1} \cdot$ Mona-Elisabeth Revheim ${ }^{1,4,5} \cdot$ Abass Alavi $^{1}$ \\ Published online: 25 January 2020 \\ (C) Springer-Verlag GmbH Germany, part of Springer Nature 2020
}

Vascular dementia is a progressive cerebrovascular disorder caused by reduced blood flow to the brain, which may lead to significant loss of brain function [1]. This cerebrovascular disease has an estimated prevalence of $1-4 \%$ of adults over age 65 , a percentage that approximately doubles every 10 years [2]. The progression of vascular dementia depends on numerous factors, including age-related risks such as hypertension, diabetes, and hyperlipidemia, which lead to the buildup of atherosclerotic plaques in the carotid arteries [3, 4]. Moreover, many cases of dementia have been reported to display both Alzheimer's disease (AD) and vascular dementia traits, and over $80 \%$ of $\mathrm{AD}$ cadavers show evidence of cerebrovascular disease [5]. A molecular imaging approach using positron emission tomography (PET) for the detection and diagnosis of atherosclerosis upstream of vascular dementia could have a substantial impact on treating an aging population suffering from cognitive impairment.

It is well-established that the brain has high metabolic demands, primarily to maintain neuronal membrane potential and ionic homeostasis. Although the brain constitutes just $2 \%$ of adult body mass, up to $20 \%$ of cardiac output is directed toward cerebral blood flow [6]. Moreover, prolonged disruption of either oxygen or glucose supply to the brain may result in irreversible neuronal cell damage in a matter of minutes [7]. Consequently, hypoxia secondary to vascular disease can lead

This article is part of the Topical Collection on Neurology

Abass Alavi

abass.alavi@pennmedicine.upenn.edu

Austin J. Borja

austin.borja@pennmedicine.upenn.edu

Emily C. Hancin

emily.hancin@temple.edu

Vincent Zhang

vincent.zhang@pennmedicine.upenn.edu

Mona-Elisabeth Revheim

monar@ous-hf.no to profound impact on cognitive function [8-11]. Prolonged cerebrovascular disease for years is considered a major cause of age-related cognitive decline [12-14]. Vascular dementia, a progressive cerebrovascular disease, is caused by reduced cerebral blood flow and possible emboli and eventually leads to significant loss of brain metabolism and function [15-17]. Individuals at the highest risk for vascular dementia are those with a recent history of stroke or transient ischemic attacks $[18,19]$. While these episodes would lead to an acute decline in cognitive function, prolonged alterations in cerebral blood flow over years or decades will also result in profound loss of cognitive function [20]. Preliminary data from our lab has showed that subjects with cardiovascular risk factors demonstrate significantly lower FDG uptake values compared with age- and sex-matched healthy controls (Fig. 1). These findings suggest an association between cardiovascular risk factors and altered global brain metabolism.

The development of vascular dementia is far from uniform. In many cases, vascular dementia is stroke-related, following either a large stroke or a series of smaller strokes [21]. This form of vascular dementia leads to long periods of normalcy, interjected with short time points of sudden decline of cognitive function [22]. In contrast, vascular dementia may be related to subcortical white matter disease due to cerebral small vessel disease, resulting in gradual cognitive impairment over

1 Department of Radiology, Hospital of the University of Pennsylvania, Philadelphia, PA, USA

2 Perelman School of Medicine at the University of Pennsylvania, Philadelphia, PA, USA

3 Lewis Katz School of Medicine at Temple University, Philadelphia, PA, USA

4 Division of Radiology and Nuclear Medicine, Oslo University Hospital, Oslo, Norway

5 Faculty of Medicine, Institute of Clinical Medicine, University of Oslo, Oslo, Norway 
many years [23]. These two vascular dementia subtypes share a key commonality: association with atherosclerosis. Bos et al. demonstrated that atherosclerosis, particularly in the extracranial carotid arteries, is not only the strongest risk factor for stroke but also a key underlying player for the development of small vessel disease [22, 24]. In this study, the authors examined the relationship between dementia risk and the vascular calcification volume in various cerebral arteries. They further concluded that the presence of atherosclerosis adversely contributed to premature cognitive degeneration prior to its transition to full-blown dementia. Therefore, these findings suggest that the early detection of atherosclerosis may play a role in preventing irreversible cognitive loss in this population.

Patterns of cerebral ${ }^{18} \mathrm{~F}$-fluorodeoxyglucose (FDG) uptake in the brain have been characterized extensively in both healthy controls and in patients with dementia, and also global uptake of this tracer has been shown to represent an excellent marker for changes in cognitive function [25-28]. Several groups have published data that support this concept. Recently, Shivamurthy et al. demonstrated the usefulness of FDG-PET/CT by highlighting distinct differences in metabolic activity between healthy brains and those of patients with dementia [26, 27, 29-31]. As such, this imaging modality is essential in assessing the consequences of atherosclerotic disease on cognition and mental performance of the affected population. Future studies should be designed to investigate the presence of a direct correlation between cardiovascular disease risk factors and cognitive function.

Atherosclerotic plaques can be identified using structural imaging modalities, including ultrasound, magnetic resonance imaging (MRI), and computed tomography (CT) [32-34]. However, these structural imaging modalities rely large plaques, which present only in late stages of disease, at which point the damage is irrversible (Fig2) [35]. In contrast, fused PET/CT imaging has demonstrated potential early diagnosis of atherosclerosis. While FDG-PET/CT is a powerful tool to assess brain function and inflammation, it has become increasingly apparent that ${ }^{18} \mathrm{~F}$-sodium fluoride $(\mathrm{NaF})$ is both a sensitive and specific tracer for the early diagnosis of atherosclerosis [36]. FDG-PET/ $\mathrm{CT}$ has been used to detect vascular inflammatory processes with variable success $[37,38]$. Blomberg et al. noted that cardiovascular disease risk factors are associated with thoracic aortic calcifications detected by NaF-PET/CT, but not with inflammation as detected by FDG-PET/CT [39]. Arani et al. demonstrated that
Fig. $1{ }^{18} \mathrm{~F}$-fluorodeoxyglucose positron emission tomography (FDG-PET/CT) images and global SUVmean of the brain displayed according to the same quantitative scale in (A) young healthy control, (B) young subject with cardiovascular risk factors, (C) old healthy control, and (D) old subject with cardiovascular risk factors, demonstrating the decrease in global cerebral metabolism in at-risk subjects

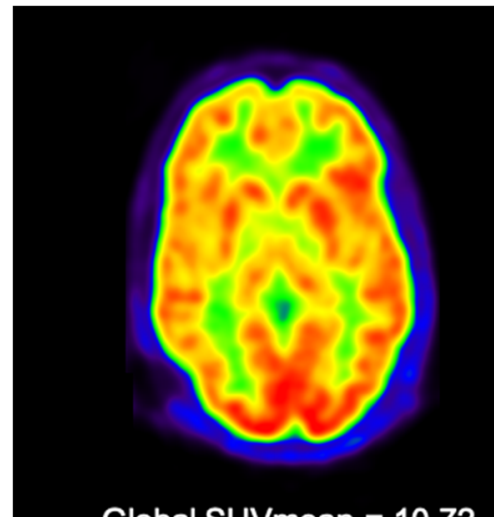

Global SUVmean $=10.72$

a

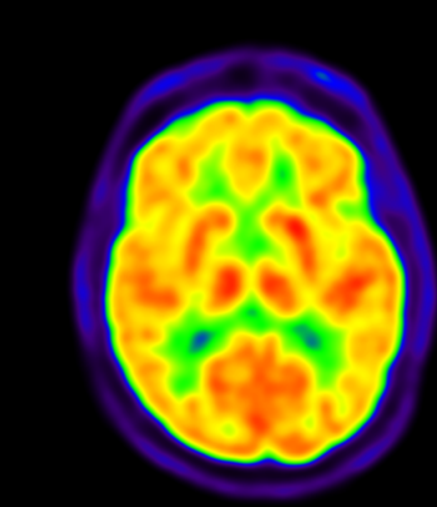

Global SUVmean $=7.20$

C

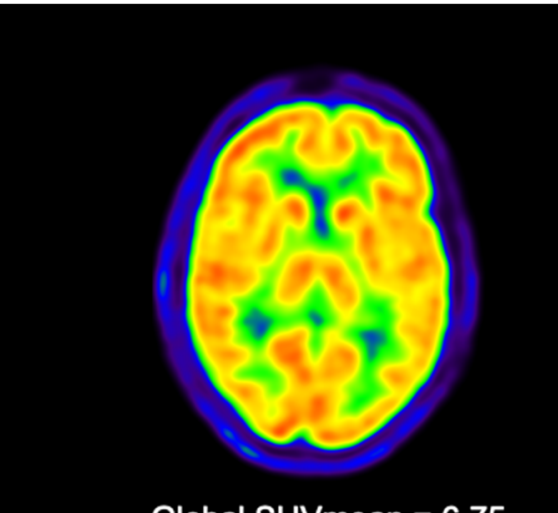

Global SUVmean $=6.75$

b

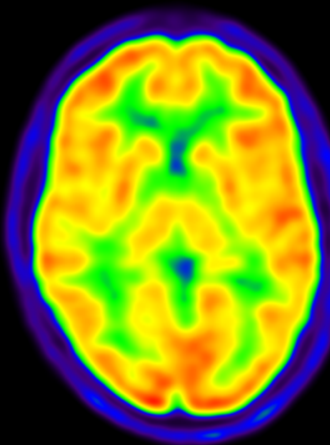

Global SUVmean $=6.70$ 
Fig. 2 The sequence of biological changes that occur during atherosclerotic vascular diseases. Atherosclerotic disease begins at the molecular and cellular level. This gradually leads to changes in blood flow and consequently functions in the brain and other organs. Finally, structural alterations occur many years following the disease process. Therefore, imaging techniques that portray early evidence for atherosclerosis, such as calcification and inflammation, may be used to diagnosis and treat the disease years before the luminal abnormalities

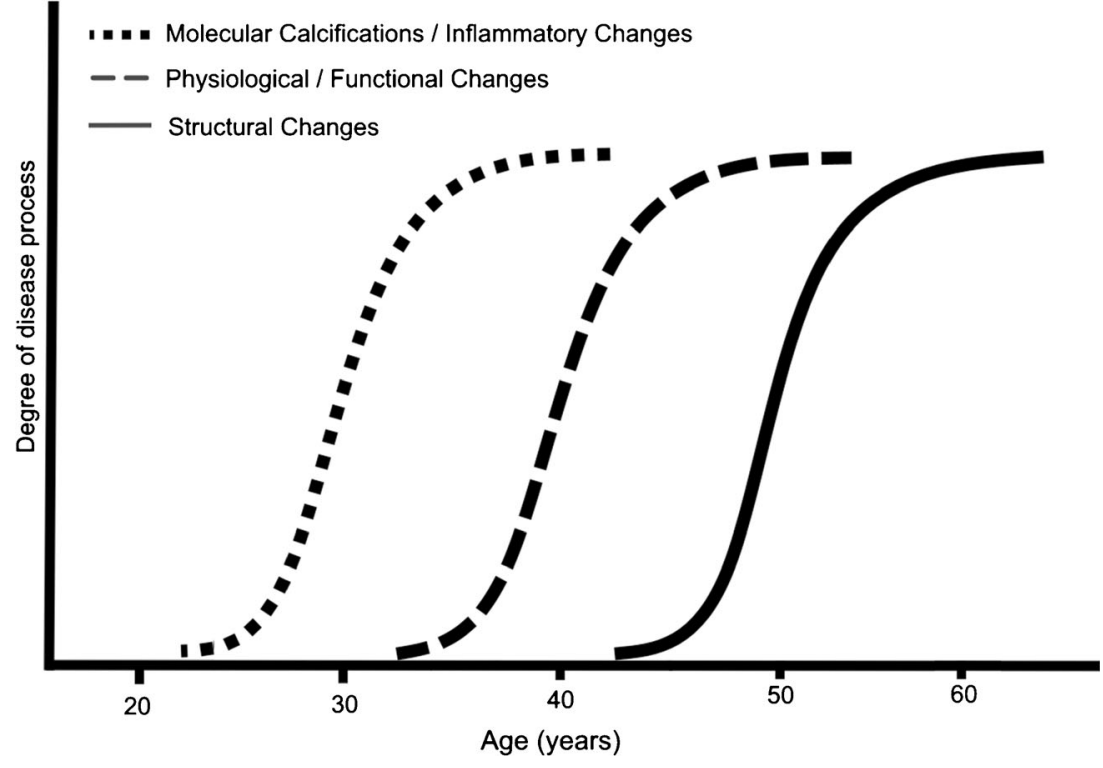

$\mathrm{NaF}$, but not FDG, uptake within the abdominal aorta significantly correlated with age and Framingham Risk Score [40, 41]. Moreover, Castro et al. showed that atherosclerotic risk factors are significantly correlated with left common carotid artery $\mathrm{NaF}$ mean and maximum standardized uptake value, revealed by NaF-PET-CT [42-45]. Based on these data, NaF-PET imaging appears to be a powerful modality to detect microcalcifications as early evidence for presence of atherosclerotic plaques.

While NaF-PET/CT may be more sensitive and specific in identifying atherosclerotic microcalcifications, FDG can be used as an effective marker to monitor brain metabolic and functional activity in this setting. As such, FDG-PET allows accurate assessment of brain function regionally and globally in patients with atherosclerotic diseases and impaired cognition and mental performance. FDG has been employed for decades as a molecular biomarker to measure metabolic consequences of several diseases and disorders [46]. We believe NaF-PET may also prove to be the tool of choice for detecting early stages of atherosclerosis when therapeutic interventions may be most effective in reversing the process. Therefore, molecular imaging techniques such as FDG- and NaF-PET/ $\mathrm{CT}$ in populations at risk for cognitive impairment may offer an exciting opportunity to detect atherosclerosis early in the disease course and treat successfully with effective therapeutic interventions.

Atherosclerotic plaques are known to lead to stroke and myocardial infarction in advanced stages of the disease [3]. Plaque formation is especially prevalent in the carotid arteries, which leads to impaired cognitive function due to luminal narrowing and micro-emboli [22]. Not surprisingly, strokes arising from atherosclerosis are considered a risk factor for the development of vascular dementia. Ivan et al. have shown that patients with past stroke history are nearly twice as likely to eventually develop vascular dementia compared to the normal populace [4]. In their analysis, the authors determined that the 10 -year risk of developing dementia in patients who had sustained their first stroke, and they determined that the occurrence of a stroke significantly increased, even in groups without genetic, sex-based, or social predispositions to the disease. These data suggest that cerebral infarcts and the underlying atherosclerosis in cerebral diseases may have longterm effects on cognitive function in the affected subjects.

Derlin et al. identified a positive correlation between cardiovascular risk factors and $\mathrm{NaF}$ uptake in the common carotid arteries in neurologically asymptomatic individuals [47]. As the carotids are the primary source of blood flow to the brain, there may be a major role for NaF-PET/CT imaging in the assessment of patients with various underlying causes of cognitive impairment and dementia. Additionally, NaF-PET/ CT may prove to be the tool of choice for detecting early stages of atherosclerosis when therapeutic interventions may prove to be effective in reversing the process.

Recent studies have demonstrated that intracranial vascular calcification is significantly correlated with cognitive impairment in both AD and vascular dementia [22, 24, 48]. Such findings precede the so-called vascular hypothesis of AD. It has therefore been hypothesized that the cognitive impairment later in life may be due to significant overlap between amyloid pathology and vascular-related brain damage [16]. Therefore, vascular dysfunction may lower the threshold of the presentation of dementia for patients with AD [21].

$\mathrm{AD}$ is a progressive neurodegenerative disorder, characterized by memory loss and cognitive decline [49]. The primary risk factor for $\mathrm{AD}$ is advanced age, but genetic factors, such as mutations affecting the amyloid precursor protein, are believed to precede early-onset cases of $\mathrm{AD}$ [50-54]. It was 
estimated that 35.6 million people lived with dementia worldwide in 2010 , with numbers expected to nearly double every 20 years, to 65.7 million by 2030 and 115.4 million by 2050 , which demonstrates a need to enhance the ongoing research to determine the causes of and potential treatments for $\mathrm{AD}$ [55]. Currently, the clinical diagnosis of $\mathrm{AD}$ is confirmed through a battery of neurocognitive tests that focus on behavioral changes and mental state assessment [56]. Yet, the earlier stages of $\mathrm{MCI}$ and preclinical $\mathrm{AD}$ often show no detectable changes in cognition. A large segment of patients with MCI patients go on to develop full AD within a matter of years [57]. Thus, there remains an unmet need for the diagnosis and treatment of early dementia due to any cause.

PET imaging has significant potential for the early diagnosis of the causes of dementia. Published data in the literature indicate that ${ }^{18} \mathrm{~F}$-florbetapir and other amyloid-targeting agents can track the pathological changes associated with $\mathrm{AD}$ prior to behavioral manifestation [58, 59]. However, recent studies have challenged the efficacy of such approaches and have emphasized the superiority of FDG-PET for accurate diagnosis of AD (Fig. 3) [60, 61]. Newberg et al. demonstrated that though both tracers have high specificity and sensitivity, FDG is the superior modality for the assessment of cognitive performance [62]. The authors determined that, while the patterns of uptake of both FDG and florbetapir revealed evidence for $\mathrm{AD}$, florbetapir does not sufficiently reflect the severity of the cognitive decline in comparison to FDG. Furthermore, multiple therapeutic trials which have used antibodies that specifically target amyloid plaques in the brain have proven to be ineffective [63, 64]. These failed attempts have demonstrated the alternate methods to monitor cognitive decline during the course of the disease [65]. Thus, FDG as a molecular probe for assessing brain glucose metabolism and $\mathrm{NaF}$ for detecting early atherosclerosis may prove to be of great importance in patients with dementia.

Cognitive impairment later in life may be due to significant association between neuronal degeneration and cerebrovascular disease [6]. Currently, drugs approved for treating AD have minimal and oftentimes unpredictable impact on the cause of the disease, and these treatments are associated with significant side effects, which lead to further deterioration of the affiliated population. Therefore, by establishing evidence for cerebrovascular disease in patients with dementia patients, clinicians may be improve and stabilize the cause of the disease. The medical community should be aware of the great potential of NaF-PET and FDG-PET in addressing this major healthcare issue affecting the aging population worldwide. We believe that future prospective and longitudinal studies should
Fig. $3{ }^{18}$ F-fluorodeoxyglucose positron emission tomography (FDG-PET/CT) and ${ }^{18} \mathrm{~F}$ -

Florbetapir images of the brain in controls (NC), mild cognitive impairment subjects (MCI), and Alzheimer's disease (AD) patients, demonstrating the superiority of FDG-PET/CT (reproduced with permission from Alavi et al. [64])

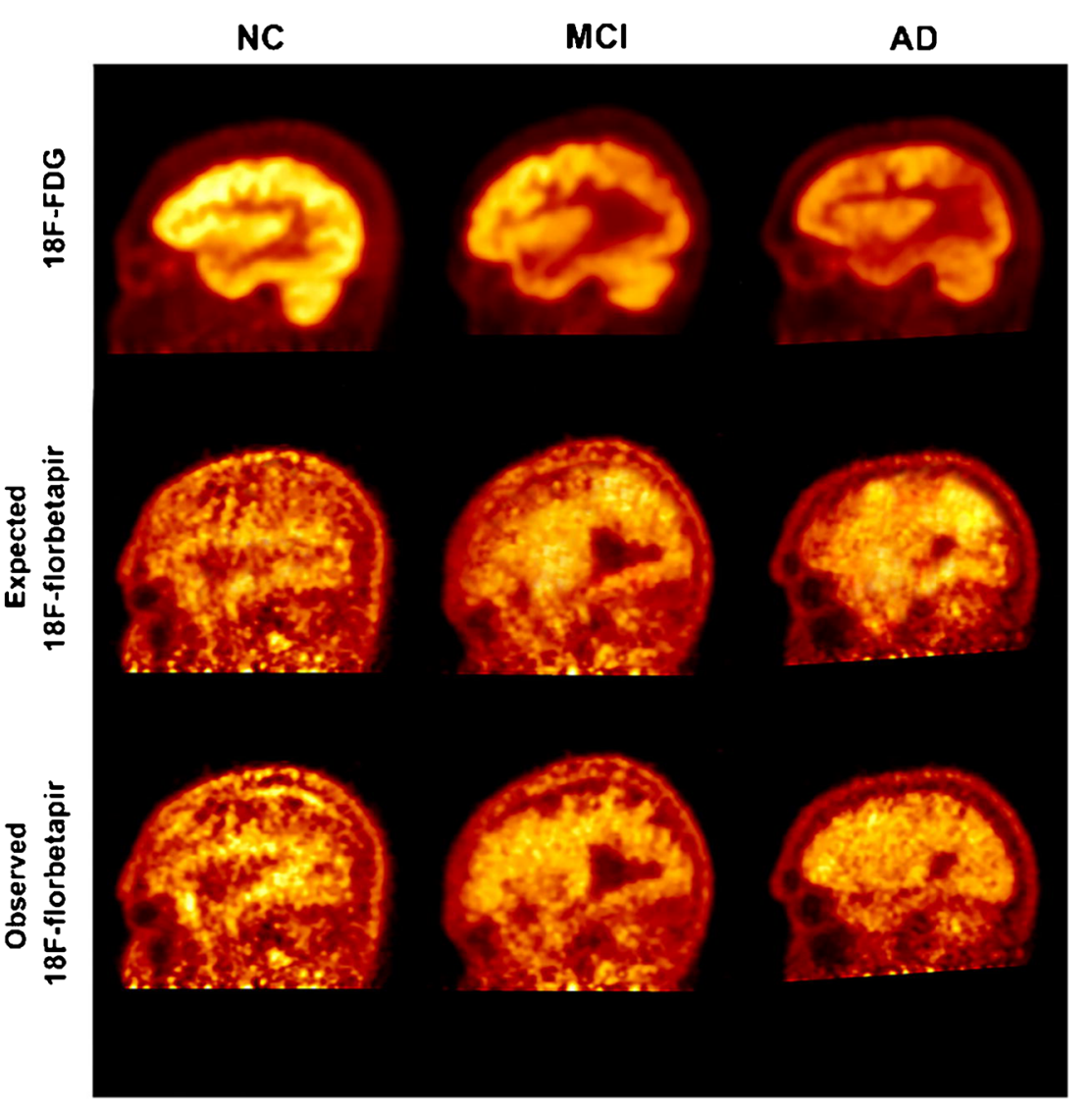


be conducted to validate the role of these powerful imaging techniques in the diagnosis and management of patients with dementia.

\section{Compliance with ethical standards}

Conflict of interest The authors declare that they have no conflict of interest.

Ethical approval This article does not contain any studies with human participants performed by any of the authors.

Statement on published images Images presented are fully anonymized and were collected with the patient's consent in the Department of Radiology, Hospital of the University of Pennsylvania, PA.

Abbreviations $\mathrm{AD}$, Alzheimer's Disease; MRI, magnetic resonance imaging; FDG, ${ }^{18} \mathrm{~F}$-fluorodeoxyglucose; NaF, ${ }^{18} \mathrm{~F}$-sodium fluoride; PET/ $\mathrm{CT}$, positron emission tomography/computed tomography

\section{References}

1. Venkat P, Chopp M, Chen J. Models and mechanisms of vascular dementia. Exp Neurol. 2015;272:97-108.

2. Baskys A, Hou AC. Vascular dementia: pharmacological treatment approaches and perspectives. Clin Interv Aging. 2007;2:327-35.

3. Benjamin EJ, Muntner P, Alonso A, Bittencourt MS, Callaway CW, Carson AP, et al. Heart disease and stroke Statistics-2019 update: a report from the American Heart Association. Circulation. 2019;139:e56-528.

4. Ivan CS, Seshadri S, Beiser A, Au R, Kase CS, Kelly-Hayes M, et al. Dementia after stroke: the Framingham study. Stroke. 2004:35:1264-8.

5. Toledo JB, Arnold SE, Raible K, Brettschneider J, Xie SX, Grossman M, et al. Contribution of cerebrovascular disease in autopsy confirmed neurodegenerative disease cases in the National Alzheimer's Coordinating Centre. Brain. 2013;136:2697-706.

6. Shabir O, Berwick J, Francis SE. Neurovascular dysfunction in vascular dementia. Alzheimer's and atherosclerosis BMC Neurosci. 2018;19:62.

7. Page S, Raut S, Al-Ahmad A. Oxygen-glucose deprivation/ reoxygenation-induced barrier disruption at the human bloodbrain barrier is partially mediated through the HIF-1 pathway. NeuroMolecular Med. 2019;21:414-31

8. Erecińska M, Silver IA. Tissue oxygen tension and brain sensitivity to hypoxia. Respir Physiol. 2001;128:263-76.

9. Gibson GE, Pulsinelli W, Blass JP, Duffy TE. Brain dysfunction in mild to moderate hypoxia. Am J Med. 1981;70:1247-54.

10. Ishige N, Pitts LH, Hashimoto T, Nishimura MC, Bartkowski HM. Effect of hypoxia on traumatic brain injury in rats: part 1: changes in neurological function, electroencephalograms, and histopathology. Neurosurgery. 1987;20:848-53.

11. Vannucci RC. Experimental biology of cerebral hypoxia-ischemia: relation to perinatal brain damage. Pediatr Res. 1990;27:317-26.

12. Balestrini S, Perozzi C, Altamura C, Vernieri F, Luzzi S, Bartolini $\mathrm{M}$, et al. Severe carotid stenosis and impaired cerebral hemodynamics can influence cognitive deterioration. Neurology. 2013;80: 2145-50.

13. Saji N, Toba K, Sakurai T. Cerebral small vessel disease and arterial stiffness: tsunami effect in the brain? Pulse Basel. 2016;3:182-9.
14. Avirame K, Lesemann A, List J, Witte AV, Schreiber SJ, Floel A. Cerebral autoregulation and brain networks in occlusive processes of the internal carotid artery. J Cereb Blood Flow Metab. 2015;35: 240-7.

15. Iadecola $\mathrm{C}$. The pathobiology of vascular dementia. Neuron. 2013;80:844-66.

16. Gorelick PB, Scuteri A, Black SE, Decarli C, Greenberg SM, Iadecola $\mathrm{C}$, et al. Vascular contributions to cognitive impairment and dementia: a statement for healthcare professionals from the American heart association/American stroke association. Stroke. 2011;42:2672-713.

17. Smith EE. Clinical presentations and epidemiology of vascular dementia. Clin Sci. 2017;131:1059-68.

18. Yoshitake T, Kiyohara Y, Kato I, Ohmura T, Iwamoto H, Nakayama $\mathrm{K}$, et al. Incidence and risk factors of vascular dementia and Alzheimer's disease in a defined elderly Japanese population: the Hisayama study. Neurology. 1995;45:1161-8.

19. Gorelick PB. Risk factors for vascular dementia and Alzheimer disease. Stroke. 2004;35:2620-2.

20. Meyer JS, Gotoh F, Akiyama M, Yoshitake S. Monitoring cerebral blood flow, oxygen, and glucose metabolism: analysis of cerebral metabolic disorder in stroke and some therapeutic trials in human volunteers. Circulation. 1967;36:197-211.

21. van den Oord SC, Sijbrands EJ, Gerrit L, van Klaveren D, van Domburg RT, van der Steen AF, et al. Carotid intima-media thickness for cardiovascular risk assessment: systematic review and meta-analysis. Atherosclerosis. 2013;228:1-11.

22. Bos D, Vernooij MW, de Bruijn RFAG, Koudstaal PJ, Hofman A, Franco $\mathrm{OH}$, et al. Atherosclerotic calcification is related to a higher risk of dementia and cognitive decline. Alzheimers Dement. 2015;11:639-647.e1.

23. Tomimoto H. Subcortical vascular dementia. Neurosci Res. 2011;71:193-9.

24. DeCarli CS. When two are worse than one: stroke and Alzheimer disease. Neurology. 2006;67:1326-7

25. Alavi A, Newberg AB, Souder E, Berlin JA. Quantitative analysis of PET and MRI data in normal aging and Alzheimer's disease: atrophy weighted total brain metabolism and absolute whole brain metabolism as reliable discriminators. J Nucl Med. 1993;34:16817.

26. van Rhijn SJ, Glosser G, de Vries JJ, Clark CM, Newberg AB, Alavi A. Visual processing impairments and decrements in regional brain activity in Alzheimer's disease. J Clin Exp Neuropsychol. 2004;26:11-23.

27. Silverman DH, Small GW, Chang CY, Lu CS, de Aburto MAK, Chen W, et al. Positron emission tomography in evaluation of dementia: regional brain metabolism and long-term outcome. JAMA. 2001;286:2120-7.

28. Høilund-Carlsen PF, Edenbrandt L, Alavi A. Global disease score (GDS) is the name of the game! Eur J Nucl Med Mol Imaging. 2019;46:1768-72.

29. Tumeh PC, Alavi A, Houseni M, Greenfield A, Chryssikos T, Newberg A, et al. Structural and functional imaging correlates for age-related changes in the brain. Semin Nucl Med. 2007;37:69-87.

30. Newberg A, Cotter A, Udeshi M, Brinkman F, Glosser G, Alavi A, et al. Brain metabolism in the cerebellum and visual cortex correlates with neuropsychological testing in patients with Alzheimer's disease. Nucl Med Commun. 2003;24:785-90.

31. Shivamurthy VKN, Tahari AK, Marcus C, Subramaniam RM Brain FDG PET and the diagnosis of dementia. AJR Am J Roentgenol 2015;204:W76-W85.

32. Steinl DC, Kaufmann BA. Ultrasound imaging for risk assessment in atherosclerosis. Int J Mol Sci. 2015;16:9749-69.

33. Kramer CM, Anderson JD. MRI of atherosclerosis: diagnosis and monitoring therapy. Expert Rev Cardiovasc Ther. 2007;5:69-80. 
34. Cohen BA, Gale JT, Mendelson DS, Mitty HA. Computer tomography demonstration of pulmonary artery calcification in Eisenmenger's syndrome. J Comput Tomogr. 1985;9:153-6.

35. McKenney-Drake ML, Moghbel MC, Paydary K, Alloosh M, Houshmand S, Moe S, et al. 18F-NaF and 18F-FDG as molecular probes in the evaluation of atherosclerosis. Eur J Nucl Med Mol Imaging. 2018;45:2190-200.

36. Høilund-Carlsen PF, Sturek M, Alavi A, Gerke O. Atherosclerosis imaging with $18 \mathrm{~F}$-sodium fluoride PET: state-of-the-art review. Eur J Nucl Med Mol Imaging. 2019.

37. Chen Y, Zhang J, Wang Y, Yuan J, Hu W. Efficacy of cholinesterase inhibitors in vascular dementia: an updated meta-analysis. Eur Neurol. 2016;75:132-41.

38. Tahara N, Imaizumi T, Virmani R, Narula J. Clinical feasibility of molecular imaging of plaque inflammation in atherosclerosis. $\mathrm{J}$ Nucl Med. 2009;50:331-4.

39. Blomberg BA, de Jong PA, Thomassen A, Lam MGE, Vach W, Olsen $\mathrm{MH}$, et al. Thoracic aorta calcification but not inflammation is associated with increased cardiovascular disease risk: results of the CAMONA study. Eur J Nucl Med Mol Imaging. 2017;44:24958.

40. Arani L, Gharavi M, Saboury B, Al-Zaghal A, Jahangiri P, Khosravi M, et al. Assessment of the role of age and cardiovascular risk factors on $18 \mathrm{~F}$-Fluorodeoxyglucose $(18 \mathrm{~F}-\mathrm{FDG})$ and $18 \mathrm{~F}$ sodium fluoride $(\mathrm{NaF})$ uptake in abdominal aortic artery. J Nucl Med. 2018;59:1539-9.

41. Ballantyne CM. Clinical Lipidology: a companion to Braunwald's heart disease. Elsevier Health Sciences. 2009.

42. Castro S, Acosta-Montenegro O, Muser D, Emamzadehfard S, Shamchi SP, Werner T, et al. Association between common carotid artery molecular calcification assessed by $18 \mathrm{~F}-\mathrm{NaF}$ PET/CT and biomarkers of vulnerable Atheromatous plaques: results from the CAMONA study. J Nucl Med. 2017;58:443-3.

43. Borja A, Werner T, Alavi A. Role of PET/CT in vascular dementia. J Nucl Med. 2019;60:1153-3.

44. Castro S, Emamzadehfard S, Muser D, Acosta-Montenegro O, Werner T, Shamchi SP, et al. Aged related differences and cardiovascular molecular calcification a $18-\mathrm{NaF}$ PET/CT quantification of vascular calcification in the common carotid artery study. J Nucl Med. 2017;58:445-5.

45. Castro S, Muser D, Acosta-Montenegro O, Emamzadehfard S, Shamchi SP, Desjardins B, et al. Common carotid artery molecular calcification assessed by $18 \mathrm{~F}-\mathrm{NaF} \mathrm{PET} / \mathrm{CT}$ is associated with increased cardiovascular disease risk: results from the CAMONA study. J Nucl Med. 2017;58:34-4.

46. Alavi A, Hess S, Werner TJ, Høilund-Carlsen PF. An update on the unparalleled impact of FDG-PET imaging on the day-to-day practice of medicine with emphasis on management of infectious/ inflammatory disorders. Eur J Nucl Med Mol Imaging. 2020;47: $18-27$.

47. Derlin T, Wisotzki C, Richter U, Apostolova I, Bannas P, Weber C, et al. In vivo imaging of mineral deposition in carotid plaque using $18 \mathrm{~F}$-sodium fluoride PET/CT: correlation with atherogenic risk factors. J Nucl Med. 2011;52:362-8.

48. McPherson DD, Hiratzka LF, Lamberth WC, Brandt B, Hunt M, Kieso RA, et al. Delineation of the extent of coronary atherosclerosis by high-frequency epicardial echocardiography. $\mathrm{N}$ Engl $\mathrm{J}$ Med. 1987;316:304-9.

49. Jack CR, Bennett DA, Blennow K, Carrillo MC, Dunn B, Haeberlein SB, et al. NIA-AA research framework: toward a biological definition of Alzheimer's disease. Alzheimers Dement. 2018;14:535-62.

50. Hansson O, Stomrud E, Vanmechelen E, Ostling S, Gustafson DR, Zetterberg $\mathrm{H}$, et al. Evaluation of plasma Abeta as predictor of
Alzheimer's disease in older individuals without dementia: a population-based study. J Alzheimers Dis. 2012;28:231-8.

51. Tarantini S, Tran CHT, Gordon GR, Ungvari Z, Csiszar A. Impaired neurovascular coupling in aging and Alzheimer's disease: contribution of astrocyte dysfunction and endothelial impairment to cognitive decline. Exp Gerontol. 2017;94:52-8.

52. Braak H, Braak E. Neuropathological stageing of Alzheimerrelated changes. Acta Neuropathol. 1991;82:239-59.

53. Kunkle BW, Grenier-Boley B, Sims R, Bis JC, Damotte V, Naj AC, et al. Genetic meta-analysis of diagnosed Alzheimer's disease identifies new risk loci and implicates $A \beta$, tau, immunity and lipid processing. Nat Genet. 2019;51:414-30.

54. Van Cauwenberghe C, Van Broeckhoven C, Sleegers K. The genetic landscape of Alzheimer disease: clinical implications and perspectives. Genet Med. 2016;18:421-30.

55. Prince M, Bryce R, Albanese E, Wimo A, Ribeiro W, Ferri CP. The global prevalence of dementia: a systematic review and metaanalysis. Alzheimers Dement. 2013:9:63-75.e2.

56. Dubois B, Feldman HH, Jacova C, Hampel H, Molinuevo JL, Blennow $\mathrm{K}$, et al. Advancing research diagnostic criteria for Alzheimer's disease: the IWG-2 criteria. Lancet Neurol. 2014;13: 614-29.

57. Michaud TL, Su D, Siahpush M, Murman DL. The risk of incident mild cognitive impairment and progression to dementia considering mild cognitive impairment subtypes. Dement Geriatr Cogn Disord Extra. 2017;7:15-29.

58. Yeo JM, Waddell B, Khan Z, Pal S. A systematic review and metaanalysis of (18)F-labeled amyloid imaging in Alzheimer's disease. Alzheimers Dement (Amst). 2015;1:5-13.

59. Clark CM, Pontecorvo MJ, Beach TG, Bedell BJ, Coleman RE, Doraiswamy PM, et al. Cerebral PET with florbetapir compared with neuropathology at autopsy for detection of neuritic amyloid$\beta$ plaques: a prospective cohort study. Lancet Neurol. 2012;11: 669-78.

60. Khosravi M, Peter J, Wintering NA, Serruya M, Shamchi SP, Werner TJ, et al. 18F-FDG is a superior Indicator of cognitive performance compared to 18F-florbetapir in Alzheimer's disease and mild cognitive impairment evaluation: a global quantitative analysis. J Alzheimers Dis. 2019;70:1197-207.

61. Martínez G, Vernooij RW, Padilla PF, Zamora J, Cosp XB, Flicker L. 18F PET with florbetapir for the early diagnosis of Alzheimer's disease dementia and other dementias in people with mild cognitive impairment (MCI). Cochrane Database Syst Rev. 2017;11: CD012216

62. Newberg AB, Arnold SE, Wintering N, Rovner BW, Alavi A. Initial clinical comparison of $18 \mathrm{~F}$-florbetapir and $18 \mathrm{~F}-\mathrm{FDG}$ PET in patients with Alzheimer disease and controls. J Nucl Med. 2012;53:902-7.

63. Kepe V, Moghbel MC, Långström B, Zaidi H, Vinters HV, Huang S-C, et al. Amyloid- $\beta$ positron emission tomography imaging probes: a critical review. J Alzheimers Dis. 2013;36:613-31.

64. Alavi A, Barrio JR, Werner TJ, Khosravi M, Newberg A, HøilundCarlsen PF. Suboptimal validity of amyloid imaging-based diagnosis and management of Alzheimer's disease: why it is time to abandon the approach. Eur J Nucl Med Mol Imaging. 2019.

65. Castellani RJ, Lee H-G, Zhu X, Nunomura A, Perry G, Smith MA. Neuropathology of Alzheimer disease: pathognomonic but not pathogenic. Acta Neuropathol. 2006;111:503-9.

Publisher's note Springer Nature remains neutral with regard to jurisdictional claims in published maps and institutional affiliations. 